\title{
Quality of Sulfadoxine-Pyrimethamine Given as Antimalarial Prophylaxis in Pregnant Women in Selected Health Facilities in Central Region of Ghana
}

\author{
Danny F. Yeboah, ${ }^{1}$ Richmond Afoakwah, ${ }^{1}$ Ekene K. Nwaefuna, ${ }^{2}$ \\ Orish Verner, ${ }^{3}$ and Johnson N. Boampong ${ }^{1}$ \\ ${ }^{1}$ Department of Biomedical and Forensic Sciences, School of Allied Health Sciences, University of Cape Coast, Cape Coast, Ghana \\ ${ }^{2}$ Biotechnology and Nuclear Agriculture Research Institute, Ghana Atomic Energy Commission, Accra, Ghana \\ ${ }^{3}$ Department of Microbiology and Immunology, School of Medicine, University of Health and Allied Sciences, Ho, Ghana
}

Correspondence should be addressed to Richmond Afoakwah; rafoakwah@ucc.edu.gh

Received 20 November 2015; Revised 27 January 2016; Accepted 31 January 2016

Academic Editor: José F. Silveira

Copyright (C) 2016 Danny F. Yeboah et al. This is an open access article distributed under the Creative Commons Attribution License, which permits unrestricted use, distribution, and reproduction in any medium, provided the original work is properly cited.

\begin{abstract}
The use of sulfadoxine-pyrimethamine (SP) as an intermittent preventive treatment (IPT) against malaria during pregnancy has become a policy in most sub-Sahara African countries and crucially depends on the efficacy of SP. This study sets out to evaluate the effectiveness of the SP given to the pregnant women in some selected health facilities in the Central Region of Ghana to prevent maternal malaria in pregnant women. A total of 543 pregnant women recruited from 7 selected health centres in Central Region of Ghana participated in the study. Parasite density of Plasmodium falciparum was determined from peripheral blood of the pregnant women using microscopy. High performance liquid chromatography (HPLC) and dissolution tester were used to determine the quality of the SP. Malaria infection was recorded in $11.2 \%$ of pregnant women who had a history of SP consumption. SP failed the dissolution test. Pregnant women who did not receive IPT-SP were $44 \%$. Low haemoglobin level was recorded in $73.5 \%$ of the pregnant women. The results indicated that SP was substandard. IPT-SP is ineffective in preventing malaria infection.
\end{abstract}

\section{Introduction}

Over 50 million pregnant women a year are exposed to malaria resulting in 2500-10 000 maternal deaths annually [1], with at least 60 percent of them in Africa. In sub-Sahara Africa, 25 million pregnant women are at risk of Plasmodium falciparum infection every year, and one in four women has evidence of placental infection at the time of delivery [2]. In high transmission areas, malaria is associated with maternal anaemia, potentially responsible for maternal death when severe, and low birth weight due to both prematurity and intrauterine growth retardation [3]. World Health Organization (WHO) policy for malaria prevention and control during pregnancy in areas of stable transmission emphasizes a preventive package of intermittent preventive treatment (IPT), insecticide-treated bed nets (ITNs), and effective case management of malaria illness and anemia.
The 2014 WHO policy update for intermittent preventive treatment in pregnancy (IPTp) recommends that doses should be delivered at each antenatal care visit after the first trimester, with a minimum of three doses received during each pregnancy [4]. The most effective drug for IPT is sulfadoxine-pyrimethamine (SP) because of its safety for use during pregnancy, effectiveness in reproductive-age women, and feasibility for use in programmes, as it can be delivered as a single-dose treatment under observation by the health worker [5]. In areas of IPTp with SP implementation, increasing resistance to SP is a growing challenge. Already in parts of Africa and Southeast Asia, the effectiveness of SP in IPTp is being threatened by increasing levels of resistance to SP [6].

Following the implementation of IPTp-SP, clinical and parasitological parameters of pregnant women attending ANC at Agogo Hospital in Ghana improved [7]. This improvement was, however, short-lived due to resistance to 
SP [8]. The usefulness of SP for IPT in countries facing moderate to high levels of SP resistance needs to be evaluated [5]. SP treatment failure occurred in pregnant women in central Ghana during 2003-2004 [9]. Treatment failure might be a result of the administration of substandard standard drugs or the development of resistance of the parasite to the drug. The purpose of this study is to determine whether IPT with SP is able to eliminate malaria parasites from the peripheral blood of pregnant women.

\section{Methods}

2.1. Study Area and Population. The study was carried out in 7 health centres in 6 selected towns in the Central Region of Ghana. The Central Region is located at the southern part of Ghana. It is bordered by the Ashanti and Eastern regions to the north, Western region to the west, Greater Accra region to the east, and the Atlantic Ocean to the south. The Central Region is situated within latitudes $6^{\circ} 15 \mathrm{~N}$ and $5^{\circ} \mathrm{N}$ and longitudes $2^{\circ} 15 \mathrm{~W}$ and $45^{\circ} \mathrm{E}$. The region is partitioned into 13 districts (Figure 1). The region has an area of about $9.826 \mathrm{~km}^{2}$ [10] and a population of about 1.6 million [11]. Central Region is made up of two ecological zones, the coastal and the forest zones. Towns from which subjects were selected are Assin Foso, Twifo Praso, and Abura Dunkwa located in the forest zone and Cape Coast, Saltpond, and Elmina in the coastal zone. The populations of the towns are as follows: Cape Coast, 82,292; Saltpond, 16,212; Elmina, 21,103; Assin Foso, 22,837; Twifo Praso, 9,011; Abura Dunkwa, 8,439 . Fishing, farming, and petty trading are the main occupation of the inhabitants. Two rainy seasons occur in the Central Region. The main wet season is from April to September and the dry season is from November to March (http://www.travel-to-discover-ghana.com/). The study was conducted in the two seasons: March in the dry season and August in the rainy season in the year 2009.

The study was carried out on pregnant women who had reported their routine monthly antenatal visit at the antenatal clinics of seven randomly selected health facilities in Central Region of Ghana. The selected facilities included Cape Coast Metropolitan Hospital, Ewim Urban Health Centre at Cape Coast, St. Francis Xavier Hospital at Assin Foso, Twifo Praso Government Hospital, Elmina Urban Health Centre, Saltpond District Hospital, and Abura Dunkwa District Hospital.

2.2. Ethical Consideration. Ethical approval was obtained from the University of Cape Coast Institutional Review Committee. Approval was also sought from the Central Regional Directorate of the Ghana Health Service before commencement of this research. The rationale of the study was explained to the subjects with the assistance of the midwives. Voluntary participation was emphasized and ensured. Participant anonymity was also ensured. Written informed consent from each consenting pregnant woman was sought before sample collection.

2.3. Participant Recruitment and Sample Collection. Participants were randomly recruited from pregnant women who had reported their routine monthly antenatal visit at the

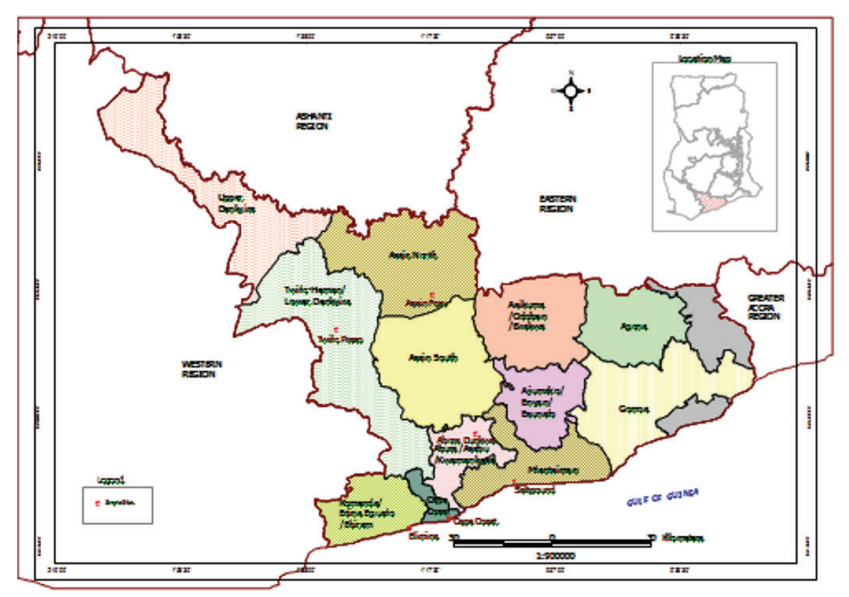

FIgURE 1: Map of Central Region of Ghana showing districts.

antenatal clinics of seven randomly selected health facilities in Central Region of Ghana. Blood was collected from participants upon their next antenatal visit from the day of recruitment. This was to ensure that blood collection was done at least a week after the consumption of SP by the pregnant women. The number of SP doses each participant has consumed was recorded. Participants were, thus, categorized as no-SP dose, one-SP dose, two-SP dose, and three-SP dose groups depending on the number of SP doses they had consumed at the point of sample collection.

Five millilitres $(5 \mathrm{~mL})$ of blood sample was collected from each participant into tubes containing EDTA by trained and licensed medical laboratory technologists in the various health facilities. All blood samples collected were stored on ice and transported to the Parasitology Research Laboratory of the Department of Biomedical and Forensic Sciences, University of Cape Coast.

2.4. Estimation of Parasite Density. Giemsa-stained thick blood films were prepared and the slides were observed under the light microscope using $\times 100$ objective lens [12]. Parasite density was determined by counting the number of malaria parasites against 200 white blood cells (WBCs). A standard WBC count of 8000 per microlitre $(\mu \mathrm{L})$ of blood is assumed for each participant. In cases where less than ten parasites were counted against 200 WBCs, counting of parasites continued until 500 WBCs were identified. A slide was considered negative if no parasite was found after counting 500 WBCs [13]. Parasite density was categorized as follows: 0 (aparasitaemic), 1-999, 1000-9999, and $\geq 10,000$ [14]. Such a classification is appropriate as higher parasite densities correspond to greater severity of the infection and acute phase of malaria [15].

2.5. Determination of Haemoglobin. The haemoglobin level of each sample collected was estimated using a Cell Dyn 1800 automated blood analyzer. A participant was considered anaemic if his/her haemoglobin level was below $11 \mathrm{~g} / \mathrm{dL}$.

2.6. Drug Sourcing. Three SP drugs were sampled for this study between October and November 2009. For the purpose 
of confidentiality, the original names and batch numbers of the drugs have been withheld. The drugs were coded as Drugs I, II, and III. Drug I is locally manufactured and given to pregnant women at the antenatal care clinics as prophylaxis. Drug II is also locally manufactured and sold on the open market in pharmacies and licensed chemical shops. Drug III is an imported product commonly found in pharmacies and licensed chemical shops. Drug I was obtained from antenatal clinics in Cape Coast, Saltpond, Elmina, Twifo Praso, Assin Foso, and Abura Dunkwa, while Drugs II and III were obtained from pharmacies and licensed chemical shops in these same areas through mystery buying. All three drugs sampled for this study had more than a year to expire. The manufacturing and expiry dates for the Drugs I were September 2007-September 2011 and those for both Drugs II and III were April 2008-April 2012. The health facilities and retail shops stored the drugs in well ventilated rooms at room temperature and kept them away from sunlight. After sampling, the drugs were stored in their various packages at room temperature until drug analysis was carried out at the laboratory of Ghana Standard Authority in February 2010.

2.7. Drug Analyses. In assessing the quality of the SP, the chemical or biological assay and the dissolution methods were employed. Reference standard solution of SP was prepared by dissolving approximately $0.5 \mathrm{~g}(500 \mathrm{mg})$ of sulfadoxine and $0.025 \mathrm{~g}(25 \mathrm{mg})$ of pyrimethamine in $10 \mathrm{~mL}$ methanol and diluted with mobile phase to $100 \mathrm{~mL}$. Five replicate injections of a system suitability preparation (standard solution) were chromatographed and the peak areas were recorded [16].

Twenty tablets of each brand of SP drug were weighed and the average weight was determined. The 20 tablets of each brand were powdered separately with mortar and pestle. An accurately weighed amount of the finely ground powder, equivalent to $0.5 \mathrm{~g}$ sulfadoxine and $0.025 \mathrm{~g}$ pyrimethamine of each sample, was dissolved in $10 \mathrm{~mL}$ of methanol with the aid of an ultrasonic bath. It was then diluted with mobile phase to $100 \mathrm{~mL}$, mixed, and filtered [16]. The samples were then introduced into an HPLC. Three replicates of each sample were run and the average peak area was recorded. The weight in milligrams of sulfadoxine and pyrimethamine in a tablet of each brand was determined. Using these results, the amounts of sulfadoxine and pyrimethamine per tablet were calculated and expressed as percentage of the label claim [17].

Tablet dissolution was performed in the ERWEKA DT 600 dissolution apparatus using 6 tablets of each product. Dissolution of the antimalarial products was carried out using 1 litre of $0.01 \mathrm{M} \mathrm{pH}$ phosphate buffer solution (sodium hydroxide and potassium dihydrogen orthophosphate, Fisher Scientific) and heated to a temperature of $37^{\circ} \mathrm{C}$, with a rotor speed of 75 revolutions per minute (rpm). The 6 tablets were introduced into the dissolution vessels and dissolution was carried for 30 minutes [18]. Samples $(2 \mathrm{~mL})$ of the dissolution media in the different vessels were withdrawn after 30 minutes and were transferred into HPLC reaction vial and then introduced into the HPLC machine for analysis for sulfadoxine and pyrimethamine content according to the HPLC method described in the USP for dissolution testing of these tablets [19].
2.8. Data Analysis. The data collected were recorded into a precoded case record forms. Thereafter, the data were transferred to Statistical Package for the Social Sciences (SPSS) version 10 for analysis. Descriptive statistics such as means and standard deviations were used to summarize quantitative variables, while categorical variables were summarized with proportions. The student $t$-test was used to compare two mean values while the one-way analysis of variance (ANOVA) was used to compare mean values in more than two groups. The Chi-square test was used to investigate associations between categorical values and also to analyze differences in proportions. For significant associations, 95\% confidence intervals were computed. A $P$ value of less than 0.05 was considered statistically significant.

\section{Results}

A total of 543 pregnant women were recruited into the programme. The ages of the participants ranged from 16 to 44 of which the majority 444 (82\%) of the women were between the ages of 18 and 34 years. The pregnancies were from 5 to 9 months. The women who were pregnant for the first time constituted 32\% (172), whilst 68\% (371) of them have had between 1 and 9 pregnancies. About $77 \%$ of the subjects were employed.

Malaria parasites were found in the peripheral blood of pregnant women who had taken SP and those who had not taken. Of the 543 pregnant women, 304 (56.0\%) had taken Drug I (SP). Those who had not taken SP were 239 (44.0\%). A total of 63 pregnant women out of the 543 (11.6\%) had malaria parasites in their blood. Out of the 63 infected pregnant women, 34 (54.0\%) had taken SP and 29 (46.0\%) had not taken SP (Table 1). There was no significant difference between those who had taken SP and were yet infected and those who had not taken SP and were infected $\left(\chi^{2}=0.27 ; P\right.$ value $=0.871$.

The haemoglobin levels of the pregnant women were generally low. As many as $399(73.5 \%)$ of the pregnant women had low haemoglobin, whilst 144 (26.5\%) had normal haemoglobin. Forty-nine pregnant women representing $77.8 \%$ of the infected pregnant women had low haemoglobin (below $11 \mathrm{~g} / \mathrm{dL}$ ), whilst $72.9 \%$ of the uninfected pregnant women also had low haemoglobin (Table 1). There was no significant difference in the haemoglobin levels of the pregnant women who had malaria parasites in their blood and those who had no malaria parasites in their blood $\left(\chi^{2}=\right.$ $0.351 ; P$ value $=0.553$ ).

There was relatively greater parasite density in the multigravidae than in the primigravidae. There were 33.9\% (184) and $66.1 \%$ (359) primigravidae and multigravidae, respectively. The infected primigravidae and multigravidae were 25 (39.7\%) and 38 (60.3\%), respectively. Forty-five infected pregnant women had parasite densities within 1-999 parasites/ $\mu \mathrm{L}$. Out of this number, 16 (35.6\%) were primigravidae compared to $29(64.4 \%)$ who were multigravidae. Eighteen of the infected pregnant women had parasite densities between 1000 and 9999 parasites/ $\mu \mathrm{L}$ and out of this number 9 $(50.0 \%)$ were primigravidae, while the remaining 9 (50.0\%) 
TABLE 1: IPTp-SP compliance and haemoglobin level against malaria in pregnant women.

\begin{tabular}{lccc}
\hline \multirow{2}{*}{ Category } & \multicolumn{2}{c}{ Malaria status } & \\
& $n(\%)$ & $n(\%)$ & \\
\hline IPTp-SP compliance & & & \\
$\quad$ Yes & $34(54.0 \%)$ & $270(56.3 \%)$ & 0.871 \\
$\quad$ No & $29(46.0 \%)$ & $210(43.7 \%)$ & \\
$\quad$ Total & $63(100 \%)$ & $480(100 \%)$ & \\
Haemoglobin level & & & \\
$\quad$ Low & $49(77.8 \%)$ & $350(72.9 \%)$ & 0.553 \\
$\quad$ Normal & $14(22.2 \%)$ & $130(27.1 \%)$ & \\
& $63(100 \%)$ & $480(100 \%)$ & \\
\hline
\end{tabular}

$P$ value based on Chi-square test.

TABle 2: Comparison of malaria parasite density between primigravidae and multigravidae.

\begin{tabular}{lccc}
\hline \multicolumn{4}{c}{ Gravidity } \\
Category & Primigravida & Multigravida & $P$ value \\
& $n(\%)$ & $n(\%)$ & \\
\hline Malaria status & & & \\
$\quad$ Infected & $25(13.6 \%)$ & $38(10.6 \%)$ & 1.000 \\
Noninfected & $159(86.4 \%)$ & $321(89.4 \%)$ & \\
Total & $184(100 \%)$ & $359(100 \%)$ & \\
Parasite density & & & \\
0 & $159(86.4 \%)$ & $321(89.4 \%)$ & \\
$1-999$ & $16(8.7 \%)$ & $29(8.1 \%)$ & 1.000 \\
$1000-9999$ & $9(4.9 \%)$ & $9(2.5 \%)$ & \\
$\geq 10000$ & $0(0 \%)$ & $0(0 \%)$ & \\
Total & $184(100 \%)$ & $359(100 \%)$ & \\
\hline
\end{tabular}

$P$ value based on Chi-square test.

were multigravidae (Table 2). The difference in the parasite densities between primigravidae and multigravidae was not significant $\left(\chi^{2}=256.0 ; P\right.$ value $\left.=1.000\right)$.

3.1. SP Drug Test. Drug I and Drug III passed the drug assay test in that the average percentage composition of both sulfadoxine and pyrimethamine in these drugs fell within the $90.0 \%-110.0 \%$ range. Drug II failed the assay test because the average percent composition of pyrimethamine in it was below 90.0 percent (Table 3). Drug dissolution test was not performed for Drug II, because it failed the first test. Drug III passed the drug dissolution test. The percentage of both sulfadoxine and pyrimethamine of Drug III dissolved in the phosphate buffer in each of the vessels was not less than 60.0 percent. Drug I failed the drug dissolution test. The percentage of pyrimethamine of Drug I dissolved in the phosphate buffer in each of the vessels was less than 60.0 percent (Table 4). Moreover, the percentage of sulfadoxine of Drug I dissolved in the phosphate buffer in two of the vessels was less than 60.0 percent.

\section{Discussion}

All participants were eligible to take SP; however, only 56\% had used IPTp-SP. This rather low compliance to IPTp-SP has been explained to be due to late first ANC clinic enrolment $[20,21]$. Irrespective of the low compliance to IPTp-SP, Plasmodium infection was comparable between participants who had consumed SP and those who had not $(P=0.553)$, suggesting the ineffectiveness of Drug I, which is used in IPTp-SP in the country. Drug failure may be due to either development of parasite resistance to the drug or inadequate drug levels, through suboptimal dosing, poor quality of the antimalarial, poor absorption, or poor metabolism to the active metabolites [22]. Drug assay and the dissolution test conducted on Drug I showed poor dissolution of the drug. Thus, the bioavailability of the drug may be compromised, leading to its ineffectiveness. This will have an implication in the spread of SP-resistant parasite strains.

Generally, the pregnant women were anaemic. Anaemia is one of the most important consequences of malaria infection during pregnancy. Anaemia during pregnancy can cause low birth weight and low birth haemoglobin in the infant. This consequently may lead to increased morbidity for the infant [23]. Relatively, there was no significant difference between the haemoglobin levels of pregnant women who had malaria infection and those who did not. This shows that irrespective of malaria, the haemoglobin levels of the pregnant women were generally low. This confirms the multifactorial etiology of anaemia in pregnancy including iron deficiency, folate deficiency, poor diet, hookworm infections, and malaria [24]. Two other known factors which contribute to development of iron deficiency anaemia in pregnancy are first the woman's iron stores at the time of conception and second the amount of iron absorbed during gestation [25]. There is a normal reduction in haemoglobin level at the beginning of pregnancy followed by a slight rise towards the end of pregnancy [26]. The initial reduction has been explained to result from increased red cell mass and demands of the foetus which exceed iron intake with consequent reduction in iron stores of the woman's body [26]. This is why iron supplementation in pregnancy has become a standard and routine practice as a preventive treatment for iron deficiency anaemia in pregnancy in developing countries [27].

In Ghana, the government provides free antenatal care. Pregnant women are given malaria prophylaxis in the form of SP tablets (Drug I) obtained from a particular pharmaceutical firm. SP from other sources are sold in pharmaceutical shops. Drug I and Drug III passed the drug assay test, implying that they contain the right quantities of the active ingredients, sulfadoxine and pyrimethamine. However, only Drug III passed the drug dissolution test. The test for dissolution determines the amount of active ingredient that is released and available for absorption. Poor manufacturing practices, poor storage of a product, and the use of incorrect excipients will lead to poor dissolution profiles [18]. The failure of Drug I to pass the drug dissolution test is an indication that a lesser amount of the drug is able to dissolve within the specified period and thus results in poor availability for absorption, rendering the drug ineffective. This situation exposes the 
TABLE 3: Calculated weight in milligram and percentage composition of sulfadoxine and pyrimethamine in Drugs I, II, and III.

\begin{tabular}{lcccc}
\hline & Weight of sulfadoxine & Percentage composition & Weight of pyrimethamine & Percentage composition \\
\hline Drug I & Per tablet $(\mathrm{mg})$ & $(\%)$ & Per tablet $(\mathrm{mg})$ & $(\%)$ \\
1 & 485.72 & 97.14 & 21.88 & 87.52 \\
2 & 490.36 & 98.07 & 23.92 & 95.68 \\
3 & 489.52 & 97.90 & 23.78 & 95.12 \\
Average & & 97.71 & & 92.76 \\
Drug II & & & & 73.56 \\
1 & 485.31 & 97.06 & 18.39 & 75.56 \\
2 & 485.88 & 97.18 & 18.89 & 76.64 \\
3 & 485.62 & 97.90 & 19.16 & 75.24 \\
Average & & 97.12 & & 90.04 \\
Drug III & 497.76 & & & 94.80 \\
1 & 498.00 & 99.55 & 22.51 & 91.40 \\
2 & 498.76 & 99.60 & 23.72 & 92.12 \\
3 & & 99.75 & 22.85 & \\
Average & & 99.63 & & \\
\hline
\end{tabular}

(mg): milligram; (\%): percentage.

TABLE 4: Recorded peak responses of five replicate injections and calculated percentage dissolution of sulfadoxine and pyrimethamine in Drugs I and III.

\begin{tabular}{ccccc}
\hline & $\begin{array}{c}\text { Average peak area } \\
\text { of sulfadoxine } \\
(\mathrm{mV})\end{array}$ & $\begin{array}{c}\text { Percentage of } \\
\text { sulfadoxine } \\
\text { dissolved }(\%)\end{array}$ & $\begin{array}{c}\text { Average peak area } \\
\text { of pyrimethamine } \\
(\mathrm{mV})\end{array}$ & $\begin{array}{c}\text { Percentage of } \\
\text { pyrimethamine } \\
\text { dissolved }(\%)\end{array}$ \\
\hline Drug I & & & 90182 & 20.20 \\
1 & 6892774 & 59.42 & 114852 & 25.74 \\
2 & 7076779 & 61.00 & 90667 & 20.31 \\
3 & 6991092 & 60.27 & 105682 & 23.67 \\
4 & 7017232 & 60.49 & 110563 & 24.76 \\
5 & 6981546 & 60.18 & 115472 & 25.86 \\
6 & 6889575 & 59.39 & 358382.5 & 80.27 \\
1 & & & 347357 & 77.80 \\
2 & 10898130 & 93.94 & 305035 & 68.32 \\
4 & 10516241 & 90.65 & 314093.5 & 70.35 \\
5 & 10790608 & 93.02 & 298327.6 & 66.82 \\
\hline
\end{tabular}

(mV): millivolts.

pregnant women to the risk of malaria infection thereby defeating the aim of IPTp in Ghana. This may account for the observed persistent malaria infection in pregnant women who have taken SP (Drug I), confirming the hypothesis that $\mathrm{SP}$ is incapable of preventing malaria in the pregnant women.

\section{Conclusion}

Drug I, being used in IPTp-SP in Ghana, is of low quality and thus may be ineffective in prevention and treatment of malaria in pregnancy.

\section{Conflict of Interests}

The authors declare that there is no conflict of interests regarding the publication of this paper.

\section{References}

[1] L. C. Morley and A. W. Taylor-Robinson, "Understanding how Plasmodium falciparum binds to the placenta and produces pathology provides a rationale for pregnancy-associated malaria vaccine development," The Open Vaccine Journal, vol. 5, no. 1, pp. 8-27, 2012. 
[2] M. Desai, F. O. ter Kuile, F. Nosten et al., "Epidemiology and burden of malaria in pregnancy," The Lancet Infectious Diseases, vol. 7, no. 2, pp. 93-104, 2007.

[3] V. Briand, G. Cottrell, A. Massougbodji, and M. Cot, "Intermittent preventive treatment for the prevention of malaria during pregnancy in high transmission areas," Malaria Journal, vol. 6, article 160, 2007.

[4] WHO, Policy Brief for the Implementation of Intermittent Preventive Treatment of Malaria in Pregnancy using SulfadoxinePyrimethamine (IPTp-SP), World Health Organization (WHO) Global Malaria Programme, WHO Department of Reproductive Health and Research, and WHO Department of Maternal, Newborn, Child and Adolescent Health, Geneva, Switzerland, 2013.

[5] WHO, "Field guide for malaria epidemic assessment and reporting-draft for field testing," WHO/HTM/MAL/2004 1097, World Health Organization, Geneva, Switzerland, 2004, http://apps.who.int/iris/bitstream/10665/68764/1/WHO_HTM_ MAL_2004.1097.pdf.

[6] A. A. Adedeji, E. Tambo, F. A. Fehintola et al., "Protective response to Sulfadoxine-pyrimethamine during intermittent presumptive treatment of malaria in pregnant women in Sagamu, Nigeria," African Journal of Pharmacy and Pharmacology, vol. 4, no. 10, pp. 754-759, 2010.

[7] F. P. Mockenhaupt, G. Bedu-Addo, C. Junge, L. Hommerich, T. A. Eggelte, and U. Bienzle, "Markers of sulfadoxinepyrimethamine-resistant Plasmodium falciparum in placenta and circulation of pregnant women," Antimicrobial Agents and Chemotherapy, vol. 51, no. 1, pp. 332-334, 2007.

[8] F. P. Mockenhaupt, G. Bedu-Addo, T. A. Eggelte et al., "Rapid increase in the prevalence of sulfadoxine-pyrimethamine resistance among Plasmodium falciparum isolated from pregnant women in Ghana," Journal of Infectious Diseases, vol. 198, no. 10, pp. 1545-1549, 2008.

[9] H. Tagbor, J. Bruce, R. Ord et al., "Comparison of the therapeutic efficacy of chloroquine and sulphadoxine-pyremethamine in children and pregnant women," Tropical Medicine \& International Health, vol. 12, no. 11, pp. 1288-1297, 2007.

[10] E. A. Opoku, Utilization of Maternal Care Services in Ghana by Region after the Implementation of the Free Maternal Care Policy, University of North Texas Health Science Center: UNTHSC Scholarly Repository, Fort Worth, Tex, USA, 2009, http://digitalcommons.hsc.unt.edu/cgi/viewcontent.cgi?article= $1077 \&$ context $=$ theses.

[11] G. O. Ovenseri-Ogbomo and V. O. Omuemu, "Prevalence of refractive error among school children in the Cape Coast Municipality, Ghana," Clinical Optometry, vol. 2, pp. 59-66, 2010.

[12] O. A. Adefioye, O. A. Adeyeba, and W. O. Hassan, "Oyeniran OA: prevalence of malaria parasite infection among pregnant women in Osogbo, Southwest Nigeria," American-Eurasian Journal of Scientific Research, vol. 2, no. 1, pp. 43-45, 2007.

[13] S. O. Coulibaly, S. Gies, and U. D’Alessandro, "Malaria burden among pregnant women living in the rural district of Boromo, Burkina Faso," American Journal of Tropical Medicine and Hygiene, vol. 77, no. 6, pp. 56-60, 2007.

[14] C. O. Obonyo, Malaria, anemia and antimalarial drug assistance in African children [Ph.D. thesis], University of Utrecht, Utrecht, the Netherlands, 2006, http://igitur-archive.library.uu.nl/dissertations/2006-0929-200211/full.pdf.
[15] M. Edison, J. B. Jeeva, and M. Singh, "Digital analysis of changes by Plasmodium vivax malaria in erythrocytes," Indian Journal of Experimental Biology, vol. 49, no. 1, pp. 11-15, 2011.

[16] H. Kaur, C. Goodman, E. Thompson et al., "A nationwide survey of the quality of antimalarials in retail outlets in Tanzania," PLoS ONE, vol. 3, no. 10, Article ID e3403, 2008.

[17] A. A. Amin and G. O. Kokwaro, "Antimalarial drug quality in Africa," Journal of Clinical Pharmacy and Therapeutics, vol. 32, no. 5, pp. 429-440, 2007.

[18] O. Onwujekwe, H. Kaur, N. Dike et al., "Quality of anti-malarial drugs provided by public and private healthcare providers in south-east Nigeria," Malaria Journal, vol. 8, article 22, 2009.

[19] WHO, The quality of antimalarials - a study in selected African countries-EDM Research Series No. 030, http://apps.who.int/ medicinedocs/pdf/s4901e/s4901e.pdf.

[20] E. K. Nwaefuna, R. Afoakwah, V. N. Orish, A. Egyir-Yawson, and J. N. Boampong, "Effectiveness of intermittent preventive treatment in pregnancy with sulphadoxine-pyrimethamine against Submicroscopic falciparum malaria in Central Region, Ghana," Journal of Parasitology Research, vol. 2015, Article ID 959427, 6 pages, 2015.

[21] J. K. Anchang-Kimbi, E. A. Achidi, T. O. Apinjoh et al., "Antenatal care visit attendance, intermittent preventive treatment during pregnancy (IPTp) and malaria parasitaemia at delivery," Malaria Journal, vol. 13, no. 1, article 162, 2014.

[22] C. H. Sibley, K. I. Barnes, and C. V. Plowe, "The rationale and plan for creating a World Antimalarial Resistance Network (WARN)," Malaria Journal, vol. 6, article 118, 2007.

[23] L. Chimsuku, F. H. Verhoeff, S. M. Maxwell et al., "The consequences of malaria infection in pregnant women and their infants," Memorias do Instituto Oswald Cruz, Rio de Janeiro, vol. 89, supplement 2, pp. 1-2, 1994.

[24] WHO, "WHO Expert Committee on Malaria: eighteenth report," WHO Technical Report Series 735, World Health Organization, Geneva, Switzerland, 1986, http://www.who.int/ iris/handle/10665/39415.

[25] L. P. McMahon, "Iron deficiency in pregnancy," Obstetric Medicine, vol. 3, no. 1, pp. 17-24, 2010.

[26] L. Reveiz, G. M. Gyte, and L. G. Cuervo, "Treatments for iron-deficiency anaemia in pregnancy," Cochrane Database of Systematic Reviews, no. 2, Article ID CD003094, 2007.

[27] K. O. Osungbade and A. O. Oladunjoye, "Preventive treatments of iron deficiency anaemia in pregnancy: a review of their effectiveness and implications for health system strengthening," Journal of Pregnancy, vol. 2012, Article ID 454601, 7 pages, 2012. 

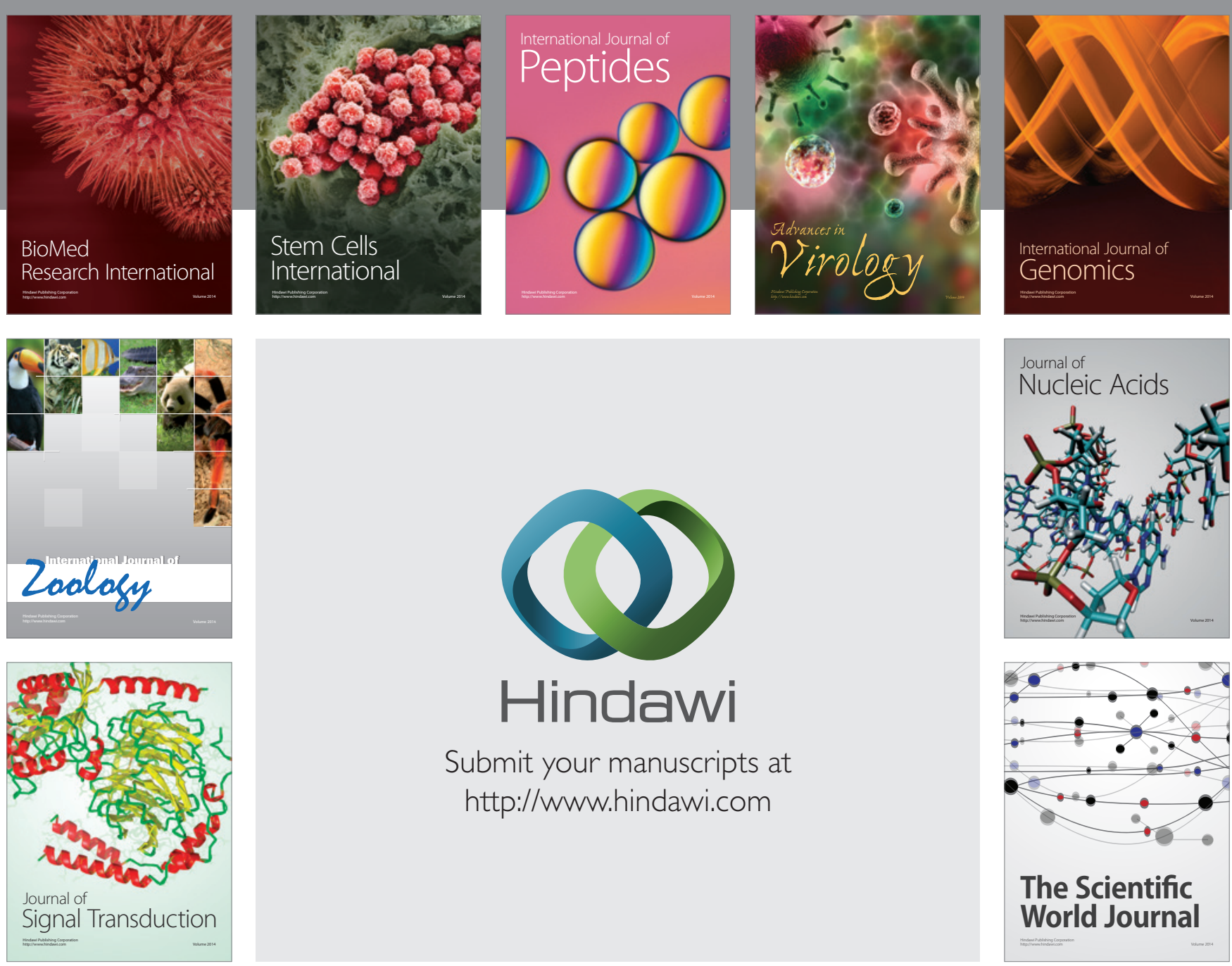

Submit your manuscripts at

http://www.hindawi.com
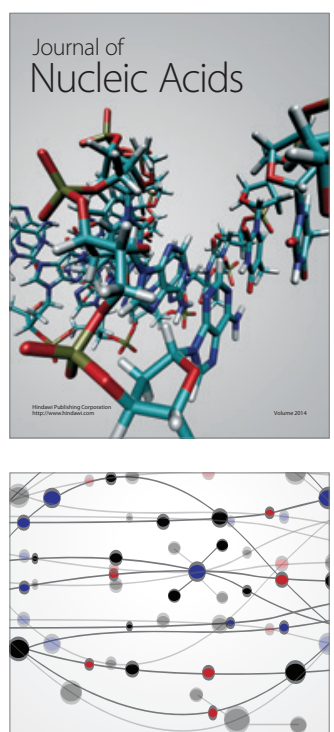

The Scientific World Journal
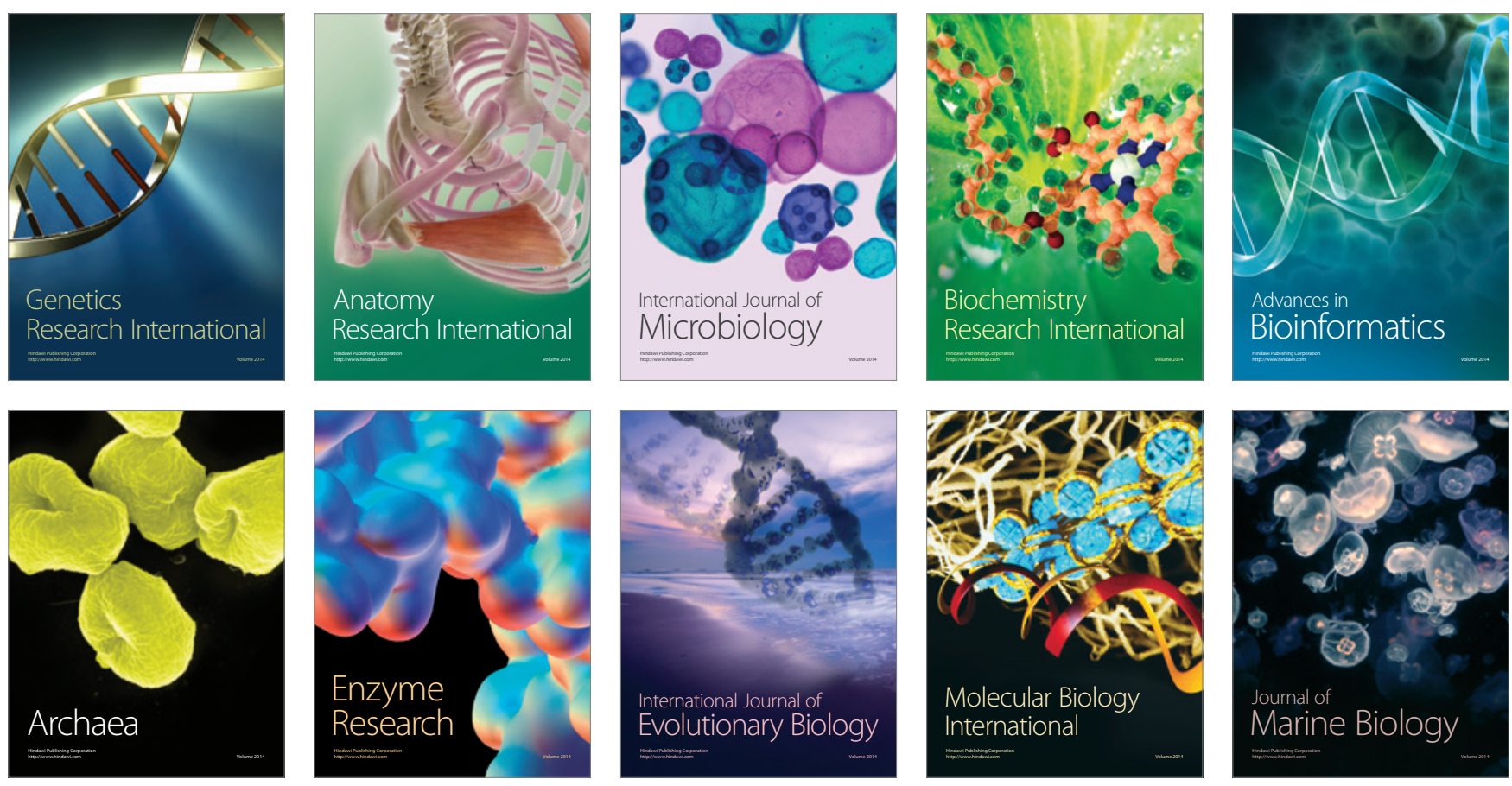